\title{
Depot injectable atorvastatin biodegradable in situ gel: development, optimization, in vitro, and in vivo evaluation
}

This article was published in the following Dove Press journal:

Drug Design, Development and Therapy

20 January 2016

Number of times this article has been viewed

\author{
Tarek A Ahmed ${ }^{1,2}$ \\ Yasser A Alharby' \\ Abdel-Rahim M El-Helw' \\ Khaled M Hosny 1,3 \\ Khalid M El-Say ${ }^{1,2}$ \\ 'Department of Pharmaceutics \\ and Industrial Pharmacy, Faculty of \\ Pharmacy, King Abdulaziz University, \\ Jeddah, Saudi Arabia; ${ }^{2}$ Department \\ of Pharmaceutics and Industrial \\ Pharmacy, Faculty of Pharmacy, \\ Al-Azhar University, Cairo, Egypt; \\ ${ }^{3}$ Department of Pharmaceutics \\ and Industrial Pharmacy, Faculty of \\ Pharmacy, Beni Suef University, Beni \\ Suef, Egypt
}

\begin{abstract}
This study aimed to develop an optimized depot injectable atorvastatin (ATR) biodegradable in situ gel (ISG) system with minimum initial burst using a central composite design. The factors selected were poly (D, L-lactide-co-glycolide) (PLGA) concentration ( $\mathrm{X}_{1}$ ), molecular weight of polyethylene glycol $(\mathrm{PEG})\left(\mathrm{X}_{2}\right)$, and PEG concentration $\left(\mathrm{X}_{3}\right)$. The independent variables were the initial burst of ATR after $2\left(\mathrm{Y}_{1}\right)$ and 24 hours $\left(\mathrm{Y}_{2}\right)$. The optimized formulation was investigated using scanning electron microscopy, Fourier transform infrared spectroscopy, and in vitro drug release in phosphate-buffered saline of $\mathrm{pH} 7.4$ for 72 hours. The in vivo pharmacokinetic study of the optimized ATR-ISG and the corresponding PEGfree ATR-ISG were conducted by intramuscular injection of a single dose $(2 \mathrm{mg} / \mathrm{kg})$ of ATR in male New Zealand White rabbits. A double-blind, randomized, parallel design was used in comparison with those of the marketed ATR tablet. Statistical analysis revealed that PLGA concentration and the molecular weight of PEG have pronounced effects on both $\mathrm{Y}_{1}$ and $\mathrm{Y}_{2}$. The optimized formulation was composed of 36.10\% PLGA, PEG 6000, and 15.69\% PEG, and exhibited characteristic in vitro release pattern with minimal initial burst. Incorporation of PEG in the formulation causes a slight decrease in the glass transition temperature value of PLGA, leading to a slight change in Fourier transform infrared spectroscopy spectrum due to possible interaction. Moreover, scanning electron microscopy photomicrograph showed smooth surface with disappearance of the cracks which characterize the surface of PEG-free formulation. The pharmacokinetic data for the optimized depot injectable ATR-ISG showed a significant $(P<0.05)$ decrease in maximum plasma concentration from 547.62 to $346.84 \mathrm{ng} /$ $\mathrm{mL}$, and increasing time to reach the maximum plasma concentration from 12 to 72 hours in comparison with the marketed tablet. The optimized ATR-ISG formulation has shown minimal initial drug burst which confirms the suitability of the ISG system in the prolongation of drug release in patients with chronic long-term therapy.
\end{abstract}

Keywords: atorvastatin, biodegradable polymers, central composite design, in situ gel

\section{Introduction}

Sustained release drug delivery systems provide several advantages over the regular formulations such as better patient comfort and compliance, prolonged drug delivery, decreased dosing frequency, and minimum side effects. ${ }^{1}$ In situ gel (ISG) system is a special class of polymeric sustained release systems that is manufactured as a liquid and solidifies after administration. ${ }^{2,3}$ It consists of a biocompatible biodegradable hydrophobic polymer that is dissolved in a water miscible/partially miscible, biocompatible solvent. ${ }^{3,4}$ The drug is dissolved or suspended in the polymeric matrix. Poly (D, L-lactide-co-glycolide) (PLGA) is one of the commonly used polymers utilized in this system. It possesses many useful properties among which are the biocompatibility,
Correspondence: Khalid M El-Say Pharmaceutical Nanotechnology Research Laboratory, Department of Pharmaceutics and Industrial Pharmacy, Faculty of Pharmacy, King Abdulaziz University, PO Box 80260, Jeddah 21589, Saudi Arabia

Tel +966 126400000

Email kelsayl@kau.edu.sa 
biodegradability, and the availability of PLGA-based marketed products, Atridox ${ }^{\circledR}$ and Eligard ${ }^{\circledR}$.

One of the major disadvantages associated with the ISG system is the initial drug burst. ${ }^{5}$ It is characterized by an initial high drug release rate at the beginning of the administration process, an effect that results in a plasma elevated drug concentration that may exceed the maximum effective concentration and may cause tissue irritation. The main cause for this phenomenon is the fast distribution of the administered drug during the sol/gel (solidification) transformation process. ${ }^{6,7}$ Other factors that could contribute to this behavior are uneven distribution of the drug inside the polymeric matrix ${ }^{8}$ and the rapid diffusion of the drug adsorbed on the surface of the polymer. ${ }^{9}$ Some efforts have been made to overcome this problem. Among these are the use of hydrophobic solvents, ${ }^{10,11}$ choice of PLGA of higher lactide rather than glycolide ratio, ${ }^{12}$ use of higher polymer concentration and molecular weight, ${ }^{13,14}$ and incorporation of plasticizer or surfactant. ${ }^{14,15}$

Atorvastatin (ATR) is a synthetic widely used cholesterollowering agent, which belongs to the drug class known as statins. Its mechanism of action involves inhibition of the enzyme 3-hydroxy-3-methyl-glutaryl-coenzyme A (HMG-CoA) reductase which catalyzes the conversion of HMG-CoA to mevalonate in the process of cholesterol biosynthesis. ${ }^{16}$ It has been reported that only $12 \%-14 \%$ of the drug is bioavailable following $40 \mathrm{mg}$ oral dose. ${ }^{17}$ The drug is subject to extensive first-pass metabolism in both the gut wall as well as in the liver cells. ${ }^{18}$ Its bioavailability is affected by parameters such as solubility, dissolution rate of the drug, gastrointestinal tract permeability, high intestinal clearance, and first-pass metabolism.

So, the aim of this study was to develop an optimized depot injectable long-term therapy of ATR formulation with a low initial drug burst using a biodegradable PLGA with different molecular weight polyethylene glycol (PEG) in different concentrations. Intramuscular administration of this formulation could be considered as an alternative for the commercially available drug oral daily tablets. This will avoid the drug peroral first pass effect, enhance the drug bioavailability, and is expected to achieve better patient comfort and compliance due to decreased drug dosing frequency.

\section{Materials and methods \\ Materials}

ATR calcium was kindly supplied as a gift sample from SAJA Pharmaceuticals Co., Ltd. (Jeddah, Kingdom of Saudi Arabia). N-methyl-2-pyrrolidone (NMP) was purchased from
Acros organics (Thermo Fisher Scientific, Waltham, MA, USA). PEG 200, 400, 1000, 4000, and 6000 were procured from Fluka AG (Buchs, Switzerland). PLGA lactide:glycolide (50:50), molecular weight 30,000-60,000 Da with intrinsic viscosity $0.45 \mathrm{dL} / \mathrm{g}$, was obtained from Sigma-Aldrich Co. (St Louis, MO, USA). All materials used were of analytical grade and were used without further modification.

\section{Methods}

\section{Preliminary study}

Preparation of PEG-free ISG formulations

Three ISG formulations using different PLGA concentrations of $20 \%, 30 \%$, and $40 \%$ were prepared. Briefly, the specified amount of polymer was dissolved in NMP in scintillation glass vials and kept shaking in a thermostatically controlled water bath shaker (Model 1031; GLF Corp., Burgwedel, Germany) at room temperature until a homogeneous clear solution was obtained. ATR in a concentration of $10 \%$ was added to the resulting polymeric solution and the vials again were kept shaking until complete dissolving of the drug.

\section{Preparation of ATR-ISG formulations \\ Utilization of central composite design}

Three different independent variables, namely, PLGA concentration $\left(\mathrm{X}_{1}\right)$, PEG molecular weight $\left(\mathrm{X}_{2}\right)$, and PEG concentration $\left(\mathrm{X}_{3}\right)$, were investigated for their effect on the initial drug release after $2\left(\mathrm{Y}_{1}\right)$ and 24 hours $\left(\mathrm{Y}_{2}\right)$. Central composite design was utilized in the development of ATRISG formulations with different levels of $\mathrm{X}_{1}, \mathrm{X}_{2}$, and $\mathrm{X}_{3}$ using Statgraphics Centurion XV version 15.2.05 software (StatPoint Technologies Inc., Warrenton, VA, USA). The design was implemented to get polynomial equations that relate the independent variables to the dependent responses. The design was performed to minimize the dependent responses $\left(\mathrm{Y}_{1}\right.$ and $\left.\mathrm{Y}_{2}\right)$. Table 1 summarizes the independent

Table I Independent and dependent variables in a central composite experimental design

\begin{tabular}{llll}
\hline Independent variables & \multicolumn{2}{l}{ Levels } \\
\hline PLGA concentration, \% $\left(\mathrm{X}_{1}\right)$ & 20 & 30 & 40 \\
Molecular weight of PEG $\left(\mathrm{X}_{2}\right)$ & $\mathrm{I}$ & 2 & 3 \\
PEG concentration, \% $\left(\mathrm{X}_{3}\right)$ & 5 & 10 & 15 \\
\hline Dependent variables & \multicolumn{2}{l}{ Constraints } \\
\cline { 2 - 4 } & Low & High & Goal \\
\hline Initial burst after 2 hours, \% $\left(\mathrm{Y}_{1}\right)$ & $\mathrm{II} .97$ & $39.6 \mathrm{I}$ & Minimize \\
Initial burst after 24 hours, \% $\left(\mathrm{Y}_{2}\right)$ & 21.32 & 49.36 & Minimize \\
\hline
\end{tabular}

Note: PEG of low molecular weight was coded I, PEG of medium molecular weight was coded 2, and PEG of high molecular weight was coded 3 .

Abbreviations: PEG, polyethylene glycol; PLGA, poly (D, L-lactide-co-glycolide). 
variables with their levels and the dependent variables with their constraints to perform the central composite design.

\section{Preparation of ATR-ISG with PEG}

Sixteen formulations were prepared by the same method described above. For low molecular weight PEG, 200 and 400 , the specified amount was added after obtaining a clear polymeric solution while for high molecular weight PEG the vials were subjected to probe sonication using Sonic

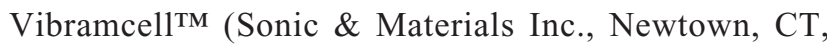
USA) under ice cooling for 30 seconds to facilitate dissolving PEG. Each formula was subjected to in vitro release study utilizing the same conditions mentioned above. The resulting composition of ATR-ISG formulations with the observed and predicted responses of these formulations is listed in Table 2.

\section{Evaluation of the prepared ATR-ISG formulations Spectroscopic evaluation of ISG ingredients}

Pure ATR, PLGA, PEG, their physical mixture, and the optimized formulation after it had been dried and crushed, which were used in the preparation of ATR-ISG, were studied for any possible interaction that may have occurred using Fourier transform infrared spectroscopy (FTIR).

Fourier transform infrared spectra were recorded on Perkin Elmer Spectrum One FTIR spectrometer (Shelton, $\mathrm{CT}$, USA) using $\mathrm{KBr}$ discs with a $2 \mathrm{~cm}^{-1}$ resolution in the range of $4,000-400 \mathrm{~cm}^{-1}$ and the spectra are displayed in Figure 1.
In vitro release study

A quantity of the formulation equivalent to $10 \mathrm{mg}$ ATR was injected into $900 \mathrm{~mL}$ phosphate-buffered saline (PBS) of pH 7.4 in a USP dissolution tester apparatus II, DT 700, Erweka $\mathrm{GmbH}$ (Heusenstamm, Germany) at $37^{\circ} \mathrm{C}$ and a rotation speed of $100 \times \mathrm{g}$. Aliquots of $2 \mathrm{~mL}$ were withdrawn at $0.5,1,2,4,6$, $8,10,12,24,48$, and 72 hours and replaced with an equivalent amount of fresh PBS to maintain a sink condition. The in vitro release study for the optimized formulation was extended for 10 days to get a complete picture about the release profile. The samples withdrawn were analyzed spectrophotometrically at $243 \mathrm{~nm}$ using Jenway $6715 \mathrm{UV}$-Visible spectrophotometer (Jenway, Stone, UK). A regression equation of a standard calibration curve was developed with the same medium to quantify the drug released. The experiment was carried out in triplicate and the release profiles of all formulations are presented in Figure 2. The release data of the optimized ATRISG formulation were analyzed using different kinetic models to designate the mechanism of drug release. ${ }^{19-23}$

\section{Characterization of the optimized ATR-ISG formulation}

The optimized ATR-ISG formulation was examined for clarity, color, and $\mathrm{pH}$. Syringeability, which is the force required to push the prepared polymeric drug solution through the syringe needle, was also evaluated using universal syringe rig (Stable Micro Systems, Surrey, UK). ${ }^{24}$ These characteristics of the optimized formulation were compared to the corresponding PEG-free formulation.

Table 2 Composition of atorvastatin in situ gel formulations and their observed and predicted responses

\begin{tabular}{|c|c|c|c|c|c|c|c|}
\hline Formulation & $\mathbf{X}_{1}(\%)$ & $\mathbf{X}_{2}$ & $\mathbf{x}_{3}(\%)$ & $\begin{array}{l}\mathbf{Y}_{1} \\
\text { Observed* }\end{array}$ & $\begin{array}{l}\mathbf{Y}_{1} \\
\text { Predicted }\end{array}$ & $\begin{array}{l}Y_{2} \\
\text { Observed* }\end{array}$ & $\begin{array}{l}\mathbf{Y}_{2} \\
\text { Predicted }\end{array}$ \\
\hline$\overline{\mathrm{FI}}$ & 20 & 4,000 & 15 & 23.55 & 23.10 & 36.84 & 36.69 \\
\hline F2 & 40 & 400 & 5 & 30.79 & 31.89 & 40.26 & 42.95 \\
\hline F3 & 40 & 4,000 & 5 & 17.24 & 18.82 & 23.55 & 26.83 \\
\hline F4 & 30 & 6,000 & 10 & 11.97 & 16.11 & 21.32 & 22.54 \\
\hline F5 & 30 & $\mathrm{I}, 000$ & 1.59 & 25.13 & 26.46 & 32.63 & 31.03 \\
\hline F6 & 40 & 400 & 15 & 14.74 & 21.69 & 27.11 & 33.09 \\
\hline F7 & 46.82 & 1,000 & 10 & 25.87 & 21.21 & 42.90 & 36.82 \\
\hline F8 & 20 & 400 & 15 & 32.90 & 31.97 & 45.92 & 45.17 \\
\hline F9 & 30 & 200 & 10 & 39.61 & 34.56 & 48.03 & 43.23 \\
\hline FIO & 20 & 4,000 & 5 & 31.45 & 25.15 & 36.05 & 32.61 \\
\hline $\mathrm{FII}$ & 20 & 400 & 5 & 35.26 & 37.17 & 44.21 & 46.42 \\
\hline $\mathrm{FI} 2$ & 13.18 & $\mathrm{I}, 000$ & 10 & 31.45 & 35.19 & 49.36 & 51.85 \\
\hline $\mathrm{FI3}$ & 40 & 4,000 & 15 & 13.03 & II.77 & 21.97 & 22.29 \\
\hline $\mathrm{FI} 4$ & 30 & 1,000 & $|8.4|$ & 18.42 & 16.17 & 28.16 & 26.17 \\
\hline FI5 & 30 & $\mathrm{I}, 000$ & 10 & 20.66 & 20.93 & 27.63 & 27.28 \\
\hline $\mathrm{FI6}$ & 30 & $\mathrm{I}, 000$ & 10 & 21.05 & 20.93 & 26.32 & 27.28 \\
\hline
\end{tabular}

Note: *The observed values of $Y_{1}$ and $Y_{2}$ represent the mean of three determinations and standard deviation $<5 \%$ of the mean.

Abbreviations: PEG, polyethylene glycol; PLGA, poly (D, L-lactide-co-glycolide); $X_{1}$, PLGA concentration; $X_{2}$, molecular weight of PEG; $X_{3}$, PEG concentration; $Y_{1}$, initial burst after 2 hours (\%); $Y_{2}$, initial burst after 24 hours (\%). 


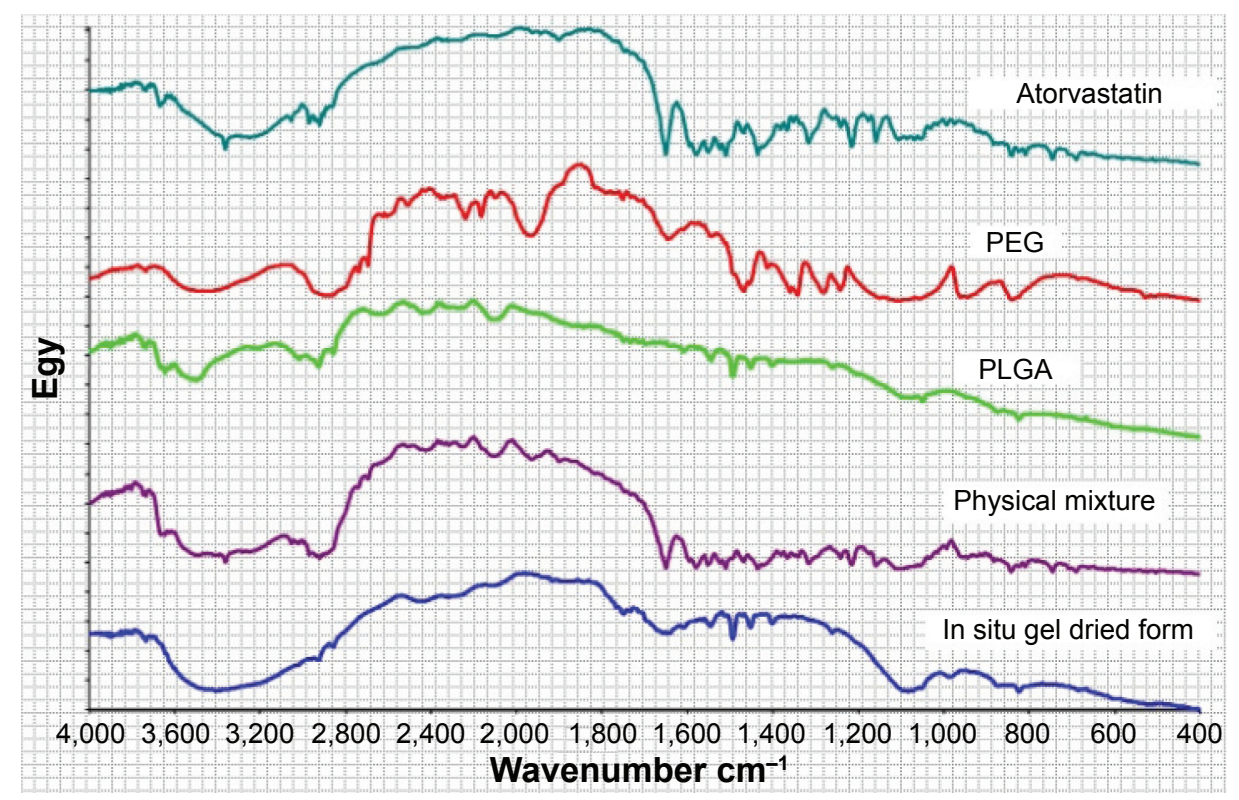

Figure I Fourier transform infrared spectra of atorvastatin, PEG, PLGA, physical mixture, and the dried in situ gel formulation. Abbreviations: PEG, polyethylene glycol; PLGA, poly (D, L-lactide-co-glycolide); Egy, energy.

\section{Surface morphology evaluation}

The surface characteristics of the optimized ATR-ISG formulation and the corresponding PEG-free ISG formulation were studied using scanning electron microscopy (SEM). Each formulation was injected separately into PBS of $\mathrm{pH} 7.4$ and kept in the buffer at $37^{\circ} \mathrm{C}$. After 24 hours the sample was collected, dried at room temperature, and subjected to characterization using SEM (Philips XL30; FEI, Hillsboro, OR, USA) and SEM photomicrographs were recorded.
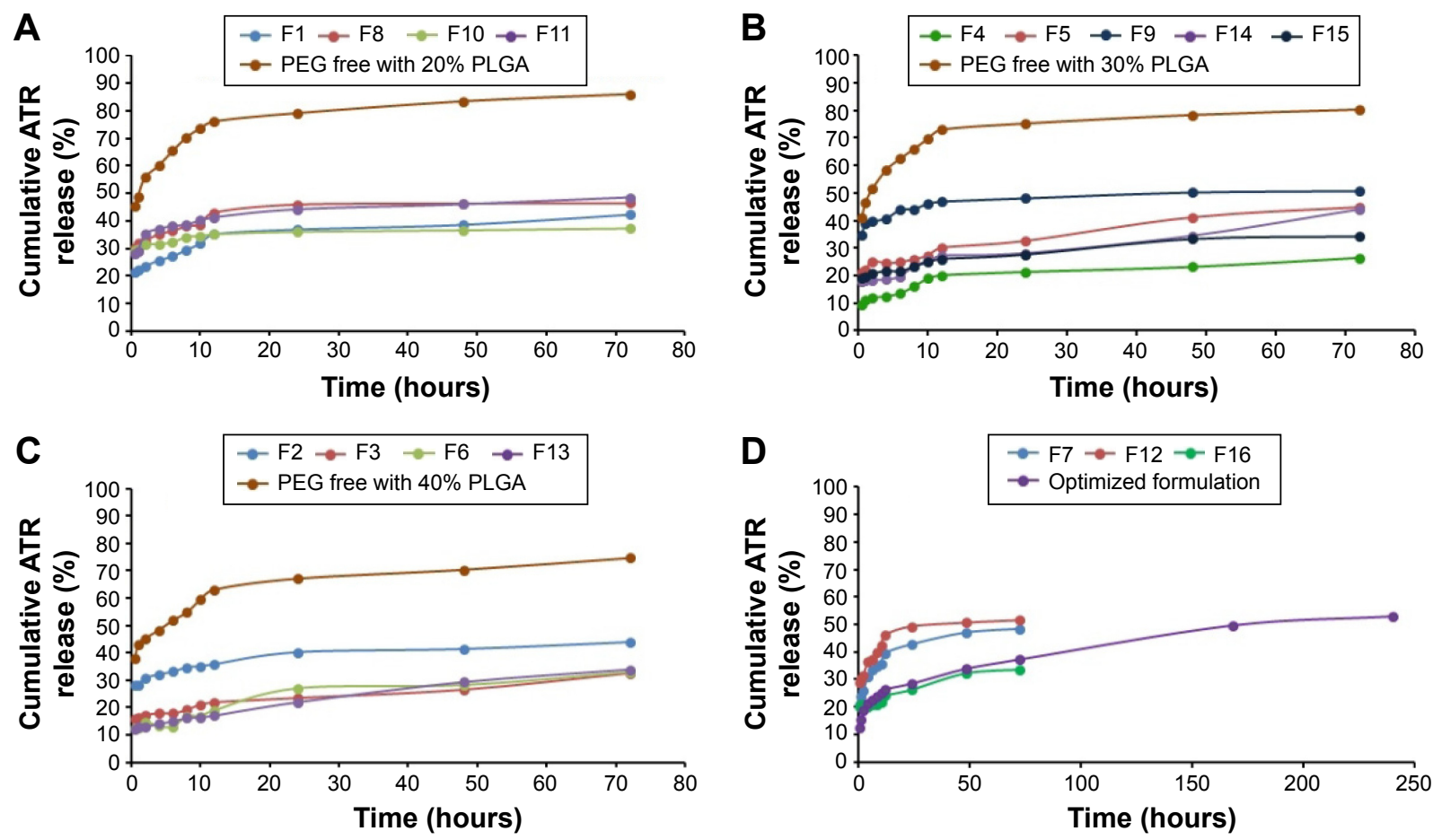

Figure 2 In vitro release profiles for ATR-ISG formulations prepared according to a central composite design.

Notes: (A) CCD formulations and the corresponding PEG-free formulation with 20\% of PLGA; (B) CCD formulations and the corresponding PEG-free formulation with $30 \%$ of PLGA; (C) CCD formulations and the corresponding PEG-free formulation with $40 \%$ of PLGA; (D) CCD formulations with different \% of PLGA and the optimized formulation. As a result of overlapping, error bars are omitted for clarity.

Abbreviations: ATR, atorvastatin; CCD, central composite design; ISG, in situ gel; PEG, polyethylene glycol; PLGA, poly (D, L-lactide-co-glycolide) acid. 


\section{In vivo and pharmacokinetic study}

Male New Zealand White rabbits with an average weight of $2.5 \mathrm{~kg}$ were provided by King Fahd Medical Research Center (Jeddah, Saudi Arabia). Animals were maintained at a temperature between $18^{\circ} \mathrm{C}$ and $22^{\circ} \mathrm{C}$ and low relative humidity (less than 30\%). The study was carried out in accordance with procedures approved by Research Ethics Committee at the Faculty of Medicine, King Abdulaziz University the local Institutional Review Board for Preclinical \& Clinical Research who ensured the care and use of experimental animals conformed to the EU Directive 2010/63/EU on the protection of animals used for scientific purposes, and Guiding Principle in Care and Use of Animals (DHEW publication NIH 80-23), and the "Principles of Laboratory Animal Care" (NIH publication \#85-23, revised in 1985). During the experiment, animals were given full access to normal standard diet and tap water ad libitum. The animals were housed two per cage in standard rabbit cages maintained at $22^{\circ} \mathrm{C} \pm 3^{\circ} \mathrm{C}$ under a 12-hour light and 12-hour dark cycle and were acclimatized at least 1 week prior to the start of the experiment. Rabbits were divided into three groups of six animals per group and kept fasted for at least 24 hours prior to the experiments. The first group received the marketed ATR tablet (reference); the second group was injected intramuscularly with the optimized ATR-ISG formulation into the right gluteus maximus muscle; while the third group was injected intramuscularly with PEG-free ISG formulation into the same muscle. The dose of ATR administered to the animals was calculated according to Reagan-Shaw et al. ${ }^{25}$ Considering the human dose of $20 \mathrm{mg}$ /day for a $70 \mathrm{~kg}$ adult, the dose for rabbits was $2 \mathrm{mg} / \mathrm{kg} /$ day. Blood samples $(0.5 \mathrm{~mL})$ were collected from the marginal ear vein at $0.5,1,2,4,8,12$, and 24 hours, and after 2, 3, 4, 5, 6, and 7 days. The collected samples were deproteinized with acetonitrile. The plasma drug concentration in each sample was calculated after analyzing the samples using ultra-performance liquid chromatography mass spectroscopy (UPLC MS/MS Acquity TQ 3100; Waters Corporation, Milford, MA, USA) equipped with ACE 5 C8 Column $(50 \times 2.1 \mathrm{~mm}, 5 \mu \mathrm{m})$. Quantification of the drug in the plasma was according to the method depicted by Gajula et $\mathrm{al}^{26}$ with slight modifications. An injection volume of $5 \mu \mathrm{L}$ was introduced into the autosampler. The mobile phase used was a mixture of $0.2 \%$ acetic acid buffer, methanol, and acetonitrile $(20: 16: 64, \mathrm{v} / \mathrm{v} / \mathrm{v})$ that was delivered at a flow rate of $1.0 \mathrm{~mL} / \mathrm{min}$ into the mass spectrometer's electrospray ionization chamber. Quantitation was achieved by MS/MS detection using an API-3200 (Applied Biosystems MDS Sciex, Concord, Canada) mass spectrometer, equipped with a turbo ion spray interface at $400^{\circ} \mathrm{C}$. The ion spray voltage was set at 3,500 V. The common parameters were nebulizer $\mathrm{N}_{2}$ gas temperature: $350^{\circ} \mathrm{C}$ and drying $\mathrm{N}_{2}$ gas flow: $200 \mathrm{~mL} / \mathrm{min}$.

\section{Pharmacokinetic analysis}

Different pharmacokinetic parameters were estimated from the obtained drug plasma concentration values using Kinetica ${ }^{\mathrm{TM}}$ software (Version 4; Thermo Fisher Scientific). Maximum plasma concentration, time to reach the maximum plasma concentration $\left(\mathrm{t}_{\max }\right)$, and mean residence time were determined. The measured drug plasma concentrations were also used to calculate the area under the plasma concentration-time curve from time zero to the last concentration time point $\left(\mathrm{AUC}_{\text {last }}\right)$ and the area under the plasma concentration-time curve from time zero to infinity $\left(\mathrm{AUC}_{\text {total }}\right)$. $\mathrm{AUC}_{\text {last }}$ was calculated according to the trapezoidal rule while $\mathrm{AUC}_{\text {total }}$ was calculated by the sum of $\mathrm{AUC}_{\text {last }}$ and the last measured concentration divided by the elimination constant $\left(\mathrm{C}_{\mathrm{t}} / \mathrm{k}_{\mathrm{e}}\right)$. The obtained data were statistically analyzed using GraphPad Prism 6 (GraphPad Software, Inc., La Jolla, CA, USA) to verify the differences between the tested groups. Two-way analysis of variance followed by Tukey's multicomparison test was used to assess the difference and a $P$-value $<0.05$ was considered to be statistically significant. In addition, the $95 \%$ confidence level is considered for testing. Each test animal was compared with the reference individually at the respective time point.

\section{Results and discussion}

To discover the optimal concentration of the used polymer (PLGA) and its effect on the initial burst of the drug from the formulation, three preliminary formulations with $20 \%$, $30 \%$, and $40 \%$ PLGA were prepared. The in vitro release of these formulations was studied for 72 hours and the release profile displayed a high initial burst release phase followed by an approximately steady state release phase (Figure 2A-C). The initial burst release stage is mainly due to the lag time between administration of the drug polymeric liquid state formulation and solidification to form the ISG system. ${ }^{6,14}$ Other possible mechanisms for this phenomenon are related to the fast release of the drug adsorbed at the surface and irregular distribution of the administered drug in the polymeric matrix. ${ }^{8,9}$ The second phase is a diffusion control release stage which is usually slower than the initial phase..$^{27,28}$

PLGA is a widely used biodegradable biocompatible polymer that hydrolyzes in the body into lactic and glycolic acid. ${ }^{7}$ It is approved by the US Food and Drug Administration as a safe compound in therapeutic formulations. ${ }^{29} \mathrm{~A}$ polymer concentration in the range of $20 \%-40 \%$ was chosen as a fast release and high initial drug burst is obtained when polymer concentration less than $20 \%$ is used. Ahmed et $\mathrm{al}^{6}$ studied the 
effect of polymer concentration on haloperidol release from PLGA in situ implant and reported a similar finding. PLGA is available in different lactide to glycolide ratios, 50:50, 65:35, $75: 25$, and $85: 15$. Polymer rich in lactide results in a highly hydrophobic polymer which degrades slowly and absorbs less water. ${ }^{30}$ High molecular weight polymer results in a polymeric solution that is difficult to inject due to its higher viscosity, while low molecular weight polymer produces a polymeric solution with a high rate of water absorption and polymer erosion. ${ }^{31}$ So, PLGA 50:50 of molecular weight $30,000-60,000 \mathrm{Da}$ with intrinsic viscosity of $0.45 \mathrm{dL} / \mathrm{g}$ in $0.1 \%$ in $\mathrm{CHCl}_{3}$ at $25^{\circ} \mathrm{C}$ was chosen as a good candidate for controlling the release of the drug in this study.

Tan et $\mathrm{al}^{15}$ have illustrated the effect of incorporation of PEG 400 into a PLGA-based ISG system. They reported a reduction in the initial burst release probably due to its plasticizing action. In another study, PEG 400 was used as a plasticizer to reduce aspirin initial burst from a PLGA-based ISG forming system. ${ }^{32}$ Ibrahim et $\mathrm{al}^{14}$ also reported a similar finding after incorporation of $10 \%-30 \%$ PEG 400 to meloxicam in situ implant formulations prepared with PLGA. The solubilizing effect of PEG could be another possible mechanism for this effect. PEG permits homogeneous distribution of the administered drug in the prepared PLGA matrix, the effect that decreases adsorption of the drug particles at the polymeric matrix. Biocompatible surfactant such as pluronics, tweens, spans, and chromophores have also been proven to influence the initial burst release from a PLGA-based ISG system. ${ }^{12,33}$ PEG could play the same role as these aforementioned surfactants. The plasticizing effect of PEG could be attributed to the decrease in the glass transition temperature of PLGA from $48^{\circ} \mathrm{C}$ to about $45.5^{\circ} \mathrm{C}$ for both PLGA-PEG 2000 and PLGA-PEG 4000 derivatives. ${ }^{34}$

Deadman et $\mathrm{al}^{35}$ defined the initial burst release as the percent of total drug released in the first 24 hours. Different researchers evaluated the drug initial burst by estimating the amount released at the first 24-48 hours. ${ }^{6,12,32,36,37}$ Other researchers determined the initial burst release at the first 2 hours. ${ }^{14}$

\section{Spectroscopic evaluation of ISG ingredients}

FTIR spectrum of pure ATR showed characteristic bands at 2,955.15, 1,313.56, 3,059.15, 1,564.97, 3,403.27, 1,656.97, 751.62 and 696.95 , and $1,104.39 \mathrm{~cm}^{-1}$ for $\mathrm{C}-\mathrm{H}$-stretching, $\mathrm{C}-\mathrm{N}$-stretching, $\mathrm{C}-\mathrm{OH}$-stretching alcoholic group, $\mathrm{C}=\mathrm{O}-$ stretching amidic group, $\mathrm{N}-\mathrm{H}$-stretching, $\mathrm{C}=\mathrm{C}$-bending, $\mathrm{C}-\mathrm{F}$-stretching, and $\mathrm{OH}-$ bending, respectively, as depicted in Figure 1. Mixing the drug with PEG in the form of physical mixture showed no considerable change in the spectrum of the pure drug while some changes in the characteristic peaks of the drug were noticed in the optimized formulation due to possible interaction of PEG with PLGA. This was obvious in the amidic group, $\mathrm{C}-\mathrm{OH}$-stretching alcoholic group, and $\mathrm{C}-\mathrm{H}$-stretching. Incorporation of $\mathrm{PEG}$ causes slight decrease in the glass transition temperature value of PLGA from $48^{\circ} \mathrm{C}$ to about $45.5^{\circ} \mathrm{C}$ as previously illustrated by Alimohammadi et al. ${ }^{34}$ Also, the plasticizing effect of PEG previously mentioned is based on the reduction of the attractive forces among the polymer chains. These effects could explain the decrease in the drug burst effect after incorporation of PEG in the formulation.

\section{Response surface methodology for optimization of ATR-ISG formulation}

A central composite design with three factors in three levels was implemented to study the influence of three different formulation parameters on ATR initial burst release after 2 $\left(\mathrm{Y}_{1}\right)$ and after 24 hours $\left(\mathrm{Y}_{2}\right)$, and evaluate the main effects, interaction effects, and quadratic effects of these factors on the dependent variables. The concentration of PLGA $\left(\mathrm{X}_{1}\right)$, molecular weight of PEG $\left(\mathrm{X}_{2}\right)$, and concentration of PEG $\left(\mathrm{X}_{3}\right)$ were selected as the independent variables.

The in vitro release profiles for the prepared formulations in comparison with PEG-free formulations are depicted in Figure 2. The drug release profile from PLGA ISG system usually exhibits a triphasic pattern: an initial fast release stage lasting for minutes or hours, followed by a slow stage of drug release lasting for days or weeks, and finally a rapid phase of drug release. ${ }^{6}$ Compared with PEG-free formulations with different concentration of PLGA depicted in Figure 2A-C, all the 16 formulations proposed by the central composite design exhibited low initial ATR release burst which confirms the impact of PEG on controlling the initial burst of ATR from the ISG formulations.

\section{Estimation of quantitative effects of the selected factors}

To estimate the quantitative effects of the selected factors, statistical analysis of central composite statistical design batches was carried out by multiple regression analysis and two-way analysis of variance using Statgraphics software. Table 3 shows the estimated effects of the selected factors, $F$-ratios, and associated $P$-values for the two responses resulting from analysis of variance.

Based on these results, $X_{1}$ had a significant antagonistic effect on both $\mathrm{Y}_{1}$ and $\mathrm{Y}_{2}$ with $P$-values $=0.032$ and 0.0156 , respectively. It was also found that $\mathrm{X}_{2}$ had a significant 
Table 3 Estimated effects of factors, F-ratio, and associated $P$-values for the initial drug release after 2 hours $\left(\mathrm{Y}_{1}\right)$ and 24 hours $\left(\mathrm{Y}_{2}\right)$

\begin{tabular}{|c|c|c|c|c|c|c|}
\hline \multirow[t]{2}{*}{ Factor } & \multicolumn{3}{|l|}{$\mathbf{Y}_{1}$} & \multicolumn{3}{|l|}{$\mathbf{Y}_{2}$} \\
\hline & Estimate & F-ratio & $P$-values & Estimate & F-ratio & $P$-values \\
\hline$X_{1}$ & -8.310 & 7.72 & $0.0320 *$ & -8.932 & 11.17 & $0.0156^{*}$ \\
\hline$X_{2}$ & -10.97 & 13.46 & $0.0105^{*}$ & -12.303 & 21.19 & $0.0037^{*}$ \\
\hline$x_{3}$ & -6.122 & 4.19 & 0.0866 & -2.891 & 1.17 & 0.3208 \\
\hline$x_{1}^{2}$ & 5.14 & 2.00 & 0.2067 & 12.058 & $13.8 \mid$ & $0.0099 *$ \\
\hline$X_{1} X_{2}$ & -0.525 & 0.02 & 0.8975 & -1.153 & 0.11 & 0.7526 \\
\hline$x_{1} x_{3}$ & -2.5 & 0.41 & 0.5459 & -4.308 & 1.52 & 0.2636 \\
\hline$X_{2}{ }^{2}$ & 3.110 & 0.73 & 0.4246 & 3.959 & 1.49 & 0.2683 \\
\hline$x_{2}^{2} x_{3}$ & 1.575 & 0.16 & 0.7008 & 2.663 & 0.58 & 0.4747 \\
\hline$x_{3}^{2}$ & 0.271 & 0.01 & 0.9429 & 0.932 & 0.08 & 0.7836 \\
\hline
\end{tabular}

Note: *Significant effect of factors on individual responses, $P<0.05$.

Abbreviations: PEG, polyethylene glycol; PLGA, poly (D, L-lactide-co-glycolide); $X_{1}$, PLGA concentration; $X_{2}$, molecular weight of PEG; $X_{3}$, PEG concentration; $Y_{1}$, initial burst after 2 hours (\%); $Y_{2}$, initial burst after 24 hours (\%); $X_{1}{ }^{2}, X_{2}^{2}$, and $X_{3}^{2}$ are the quadratic terms for the factors; $X_{1} X_{2}, X_{1} X_{3}$, and $X_{2} X_{3}$ are the interaction terms between the factors.

antagonistic effect on both responses $\left(P=0.0105\right.$ for $Y_{1}$ and $P=0.0037$ for $\left.\mathrm{Y}_{2}\right)$. On the other hand, $\mathrm{X}_{3}$ had no significant effect on both $\mathrm{Y}_{1}$ and $\mathrm{Y}_{2}$. In addition, $\mathrm{Y}_{2}$ was significantly affected by the synergistic effects of the quadratic term of $\mathrm{X}_{1}(P=0.0099)$. Finally, it was found that the interaction terms of $X_{1} X_{2}, X_{1} X_{3}$, and $X_{2} X_{3}$ and the quadratic term of $X_{2}$ and $X_{3}$ had no significant effect on the studied responses.

Statistical analysis and mathematical modeling of experimental data

Analysis of variance for the obtained results revealed that only the PLGA concentration $\left(\mathrm{X}_{1}\right)$ and molecular weight of PEG $\left(\mathrm{X}_{2}\right)$ had a significant pronounced effect $(P$-value $<0.05)$ on the initial burst after 2 hours and after 24 hours. This effect was displayed in the standardized Pareto charts for $\mathrm{Y}_{1}$ and $\mathrm{Y}_{2}$ (Figure 3A and B).

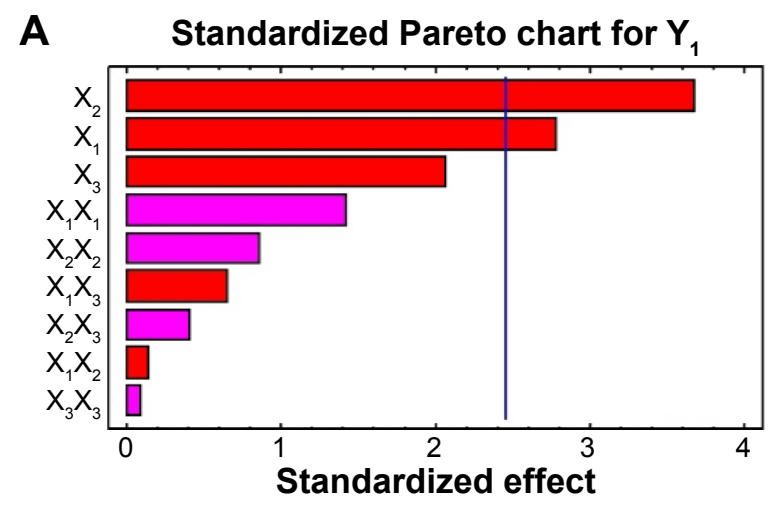

On the analysis of the obtained values for the responses regarding initial burst after 2 hours $\left(\mathrm{Y}_{1}\right)$ and initial burst after 24 hours $\left(\mathrm{Y}_{2}\right)$, the mathematical model for each response was generated and is shown in Equations 1 and 2.

$$
\begin{aligned}
\mathrm{Y}_{1}= & 74.456-1.655 \mathrm{X}_{1}-12.493 \mathrm{X}_{2}-0.286 \mathrm{X}_{3}+0.026 \mathrm{X}_{1}^{2} \\
& -0.026 \mathrm{X}_{1} \mathrm{X}_{2}-0.025 \mathrm{X}_{1} \mathrm{X}_{3}+1.555 \mathrm{X}_{2}^{2}+0.158 \mathrm{X}_{2} \mathrm{X}_{3} \\
& +0.005 \mathrm{X}_{3}^{2} \\
\mathrm{Y}_{2}= & 108.866-3.518 \mathrm{X}_{1}-15.002 \mathrm{X}_{2}+0.098 \mathrm{X}_{3}+0.06 \mathrm{X}_{1}^{2} \\
& -0.058 \mathrm{X}_{1} \mathrm{X}_{2}-0.043 \mathrm{X}_{1} \mathrm{X}_{3}+1.979 \mathrm{X}_{2}^{2}+0.266 \mathrm{X}_{2} \mathrm{X}_{3} \\
& +0.019 \mathrm{X}_{3}^{2}
\end{aligned}
$$

These equations imitate the quantitative influence of the formulation variables on $\mathrm{Y}_{1}$ and $\mathrm{Y}_{2}$ responses. By increasing the concentration of PLGA and using PEG with high molecular weight, the initial burst after 2 hours $\left(\mathrm{Y}_{1}\right)$ and after 24 hours $\left(\mathrm{Y}_{2}\right)$ was decreased as illustrated in the response surface plot in Figure 4.

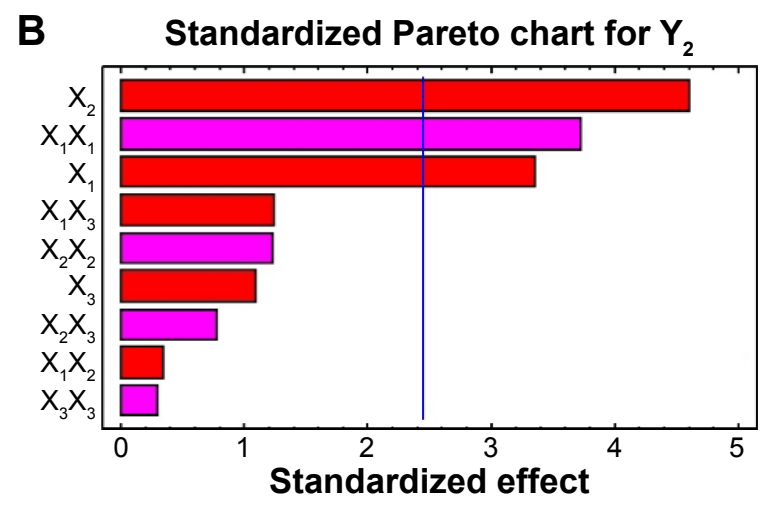

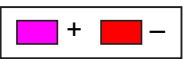

Figure 3 Standardized Pareto charts for the effect of the studied variables on $Y_{1}$ and $Y_{2}$.

Notes: Standardized Pareto chart for initial burst at 2 hours (\%) (A). Standardized Pareto chart for initial burst at 24 hours (\%) (B).

Abbreviations: PEG, polyethylene glycol; PLGA, poly (D, L-lactide-co-glycolide); $X_{1}$, PLGA concentration; $X_{2}$, molecular weight of PEG; $X_{3}$, concentration of PEG; $Y_{1}$, initial burst at 2 hours (\%); $Y_{2}$, initial burst at 24 hours (\%); $X_{1} X_{1}, X_{2} X_{2}$, and $X_{3} X_{3}$ are the quadratic terms for the factors; $X_{1} X_{2}, X_{1} X_{3}$, and $X_{2} X_{3}$ are the interaction terms between the factors. 

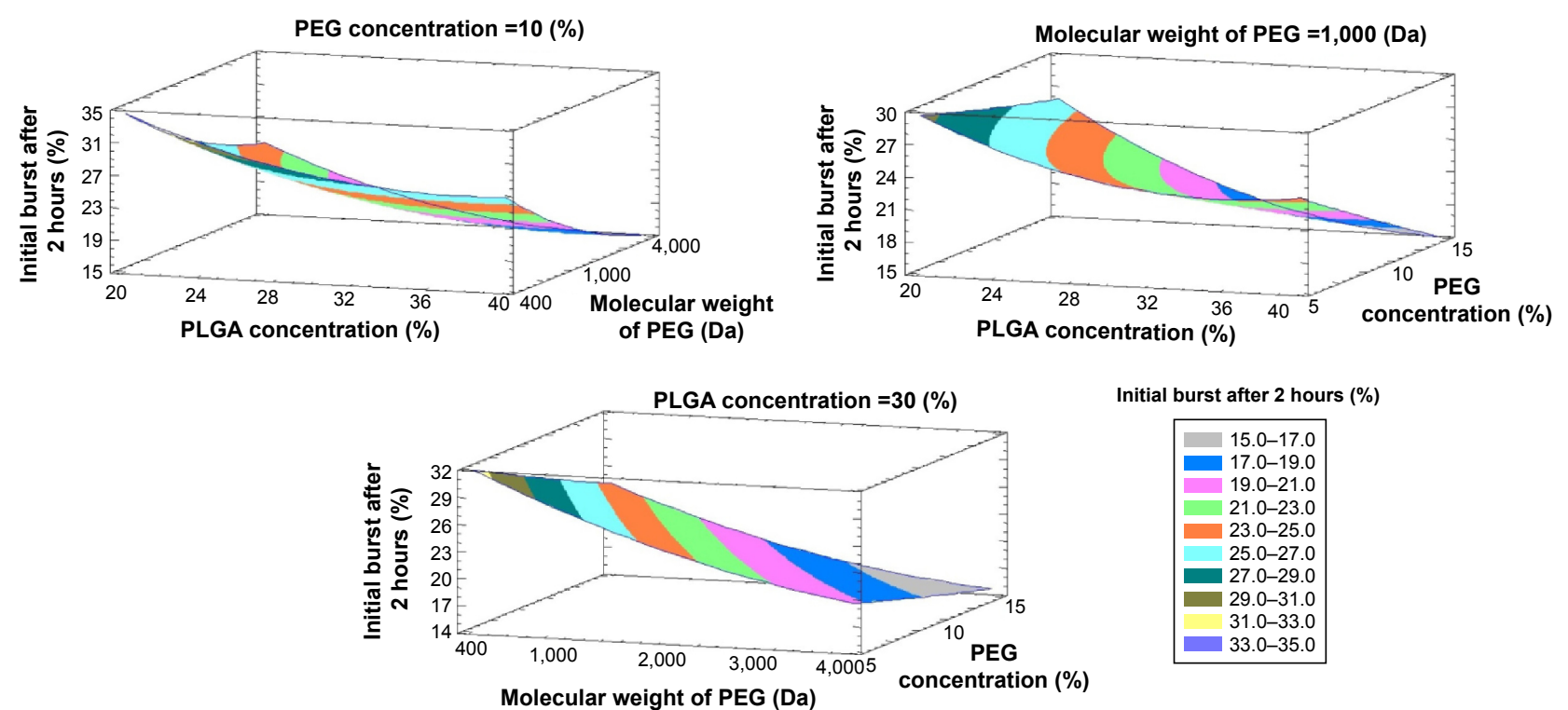

Initial burst after 2 hours (\%)

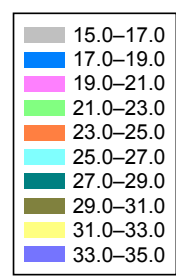

Molecular weight of PEG (Da)
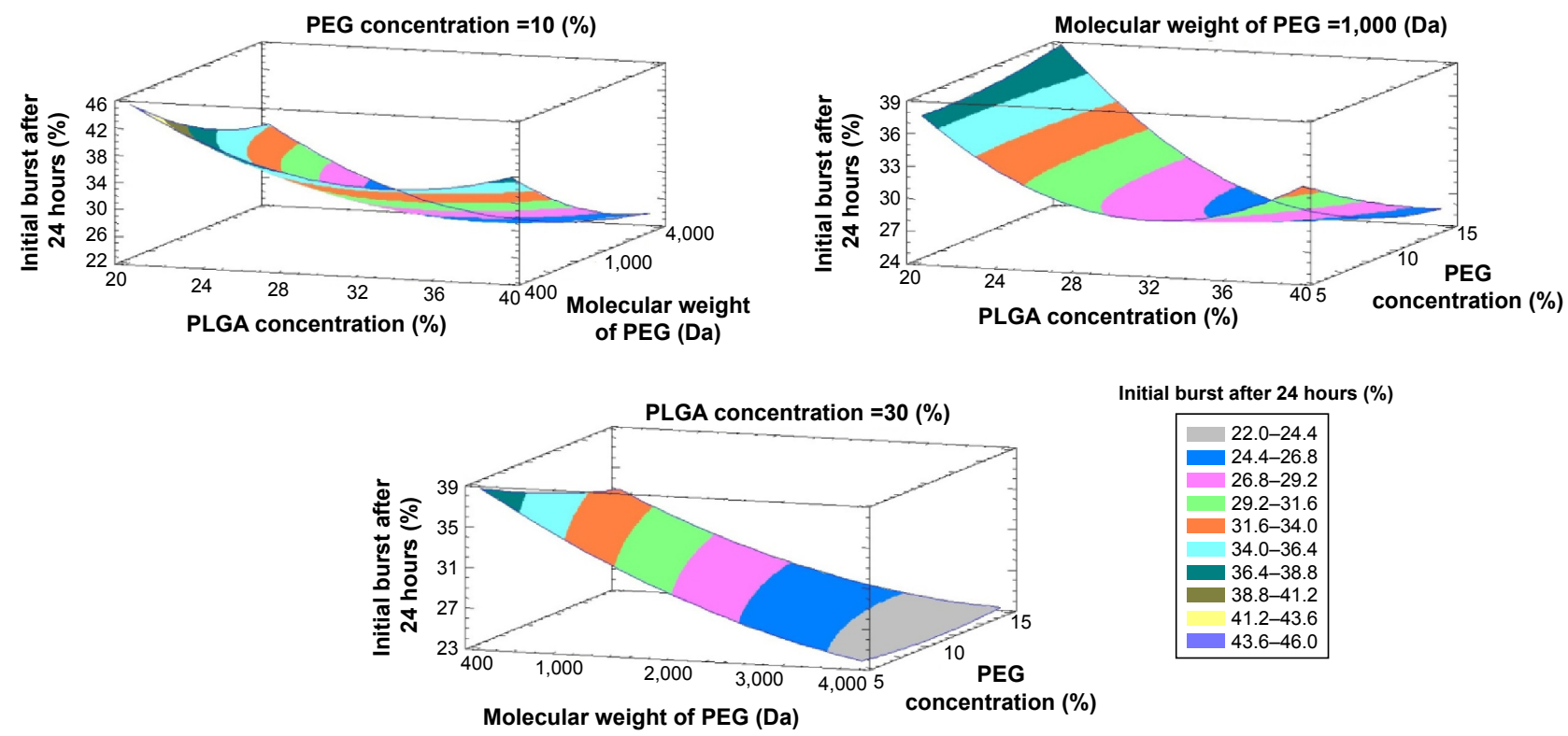

Initial burst after 24 hours (\%)

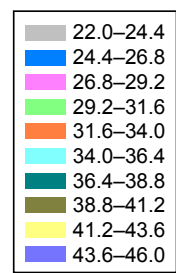

Figure 4 Estimated response surfaces with contour plots (three-dimensional) showing the effect of the studied variables on $Y_{1}$ and $Y_{2}$

Abbreviations: PEG, polyethylene glycol; PLGA, poly (D, L-lactide-co-glycolide); $Y_{1}$, initial burst at 2 hours (\%); $Y_{2}$, initial burst at 24 hours (\%).

\section{Optimization of the formulation variables}

To develop an optimized ATR-ISG formulation with lower $\mathrm{Y}_{1}$ and $\mathrm{Y}_{2}$ values of $11.70 \%$ and $21.12 \%$ respectively, the multiple response optimization was performed using Statgraphics software. According to this optimization process, the predicted values of $\mathrm{X}_{1}, \mathrm{X}_{2}$, and $\mathrm{X}_{3}$ at maximum desirability of the responses that fulfill the requirement of optimized formulation were $36.10 \%$, PEG 6000 , and $15.69 \%$, respectively. This optimized formulation was prepared and evaluated to verify the validity of the model. The predicted model values for $Y_{1}$ and $Y_{2}$ were $15.7 \%$ and $25.15 \%$, respectively, while the observed values for $\mathrm{Y}_{1}$ and $\mathrm{Y}_{2}$ were $18.75 \%$ and $28.52 \%$, respectively (Figure 2D), which seem to be very close to the predicted ones that indicate validity of the model.

\section{Characterization of the optimized formulation}

The PEG-free formulation was clear and of yellowish brown color. This color is mainly attributed to the color of PLGA polymer since the solvent used, NMP, is a colorless liquid. The $\mathrm{pH}$ was almost neutral and exhibited a value of 7.54 which is approximately the $\mathrm{pH}$ value of NMP of 7.7-8 as previously reported. ${ }^{24}$ Incorporation of PEG in the optimized formulation does not significantly affect the clarity, color, or 

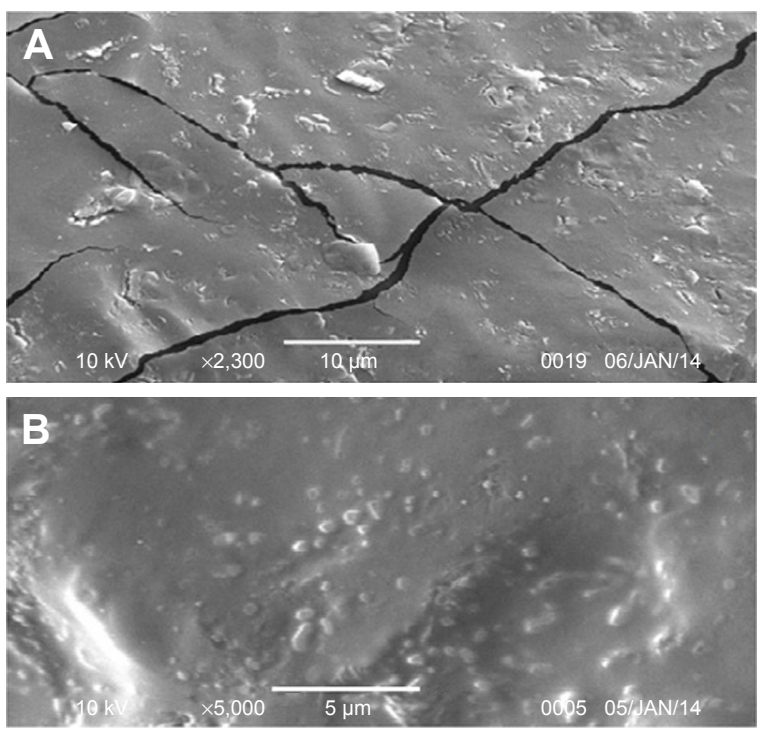

Figure 5 Scanning electron microscopy photomicrographs for ATR-ISG surface: (A) PEG-free formulation and (B) the optimized ATR-ISG formulation.

Abbreviations: ATR-ISG, atorvastatin in situ gel; PEG, polyethylene glycol.

even the $\mathrm{pH}$. It was noticed that the viscosity of the prepared ISG formulation slightly increased after incorporation of the specified quantity of PEG. This was confirmed by measuring the syringeability which was slightly reduced and resulted in a slightly higher force required to push the syringe plunger. Syringeability of both formulations, optimized and PEG-free, was within the acceptable range as previously mentioned. ${ }^{24}$

SEM photomicrographs of the optimized ATR-ISG formulation and the corresponding PEG-free ISG formulation are illustrated in Figure 5. As is clear from the figure, PEG-free ISG formulation showed a cracked surface (Figure 5A) whereas the incorporation of PEG in the optimized formulation leads to disappearance of these cracks as indicated by the smooth surface (Figure 5B). This finding assumes successfulness of the technique and the benefit of incorporation of PEG in reducing the drug burst effect due to decrease in the glass transition temperature of PLGA with subsequent pronounced plasticizing action as previously illustrated.

\section{Kinetic analysis of in vitro release data}

Kinetic analysis for the studied ISG formulations proposed by the central composite design and the optimized formulation revealed that all the studied ISG formulations were in concordance with the Higuchi diffusion model (data not shown). Previous studies indicate that Higuchi diffusion model is used to describe the drug dissolution and release from different types of modified release dosage forms such as transdermal and matrix type tablets. ${ }^{21,38,39}$ Such behavior may also be expected for the release kinetics of ATR ISG system during the release behavior of the drug from PLGA in phosphate-buffered saline (PBS) exhibited Fickian diffusion mechanism. The calculated $\mathrm{n}$ values were less than 0.5 indicating case I transport, Fickian diffusion mechanism.

\section{In vivo and pharmacokinetic study in rabbits}

The plasma concentration versus time curve is shown in Figure 6 and the pharmacokinetic parameters are listed in Table 4.

The maximum plasma concentration values reached $547.62,367.47$, and $346.84 \mathrm{ng} / \mathrm{mL}$ after oral administration of ATR tablet and intramuscular injection of both PEG-free ATR-ISG formulation and optimized ATR-ISG formulation, respectively. The $t_{\max }$ values were 12,48 , and 72 hours for oral ATR tablet, PEG-free ATR-ISG formulation, and the optimized ATR-ISG formulation, respectively, while the mean residence time values were $41.421,62.866$, and 79.652 hours for the same groups, respectively. The area under the time-concentration curve total for the optimized ATR-ISG formulation was found to be $35,932 \mathrm{ng} / \mathrm{mL} \cdot$ hour, which was significantly $(P<0.05)$ greater than that of the marketed ATR tablet $(29,185.6 \mathrm{ng} / \mathrm{mL} \cdot$ hour$)$. These results are in good agreement with the observed values for $\mathrm{t}_{\max }$ and mean residence time. From the pharmacokinetic data obtained, it was found that the optimized ATR-ISG formulation has lowered the initial burst, extended the $t_{\max }$, increased the mean residence

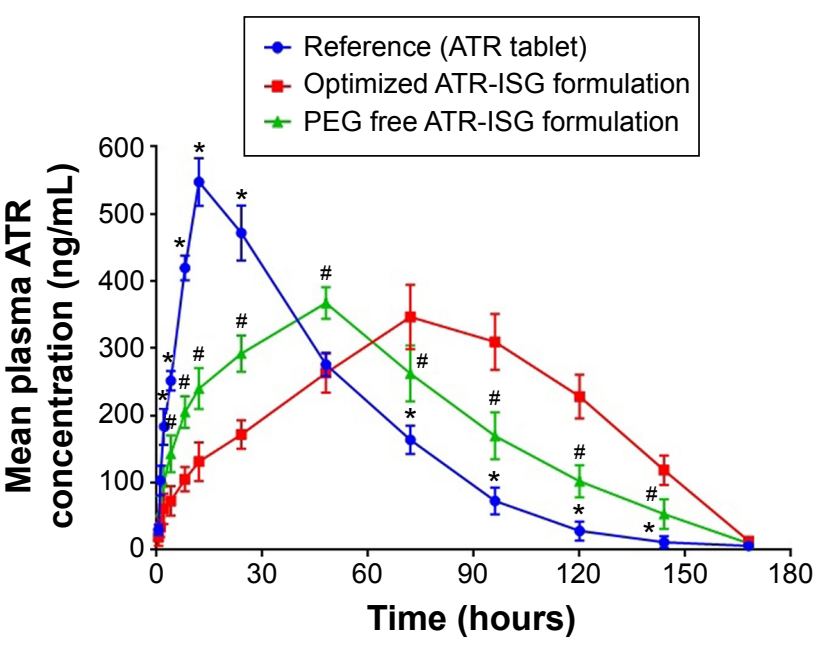

Figure 6 Plasma concentration versus time curve of ATR following the intramuscular injection of optimized ATR-ISG formulation and its corresponding PEG-free ISG system in comparison with the oral administration of the same dose of marketed tablet.

Notes: The data represent the mean \pm standard deviation $(n=6)$. *Significant difference between the optimized ATR-ISG formulation and the reference ATR tablet $(P<0.000 \mathrm{I})$; \#Significant difference between the optimized ATR-ISG formulation and the corresponding PEG-free ISG formulation $(P<0.000$ I).

Abbreviations: ATR, atorvastatin; ISG, in situ gel; PEG, polyethylene glycol. 
Table 4 Pharmacokinetic parameters after the intramuscular injection of optimized ATR-ISG and PEG-free ATR-ISG in comparison with the oral administration of a single dose $(2.5 \mathrm{mg} / \mathrm{kg})$ of the marketed ATR tablet

\begin{tabular}{llll}
\hline $\begin{array}{l}\text { Pharmacokinetic } \\
\text { parameters }\end{array}$ & $\begin{array}{l}\text { Marketed oral } \\
\text { ATR tablet }\end{array}$ & $\begin{array}{l}\text { PEG-free ATR-ISG } \\
\text { formulation }\end{array}$ & $\begin{array}{l}\text { Optimized ATR-ISG } \\
\text { formulation }\end{array}$ \\
\hline$C_{\text {max }}(\mathrm{ng} / \mathrm{mL})$ & 547.62 & 367.47 & 346.84 \\
$T_{\max }(\mathrm{h})$ & 12 & 48 & 72 \\
$A U C_{\text {last }}(\mathrm{ng} / \mathrm{mL} \cdot \mathrm{h})$ & $29,025.9$ & 31,699 & $35,635.6$ \\
$A U C_{\text {extra }}(\mathrm{ng} / \mathrm{mL} \cdot \mathrm{h})$ & 159.781 & 492.349 & 296.343 \\
$A U C_{\text {total }}(\mathrm{ng} / \mathrm{mL} \cdot \mathrm{h})$ & $29,185.6$ & $32,191.4$ & 35,932 \\
$M R T(\mathrm{~h})$ & 41.4206 & 62.8655 & 79.6521 \\
$A U M C_{\text {last }}\left(\mathrm{ng} / \mathrm{mL} \cdot \mathrm{h}^{2}\right)$ & $1.177 \times 10^{6}$ & $1.926 \times 10^{6}$ & $2.807 \times 10^{6}$ \\
AUMC $_{\text {extra }}\left(\mathrm{ng} / \mathrm{mL} \cdot \mathrm{h}^{2}\right)$ & $31,407.1$ & 97,956 & $54,819.5$ \\
\hline
\end{tabular}

Note: Data represent the mean value of six determinations.

Abbreviations: ATR, atorvastatin; AUC, area under the time-concentration curve; $\mathrm{C}_{\text {max }}$, maximum plasma concentration; ISG, in situ gel; PEG, polyethylene glycol; MRT, mean residence time; $T_{\text {max }}$, time to reach $C_{\text {max }}$. $A \cup C_{\text {last }}$, area under the time-concentration curve from time zero to 7 days; $A U C_{\text {extra }}$, area under the time-concentration curve from time 7 days to infinity; $A \cup C_{\text {total }}$, area under the time-concentration curve from time zero to infinity; $A \cup M C$, area under the moment curve.

time of ATR in the plasma, and exhibited larger area under the curve. This marked difference is attributed to the incorporation of PEG in the optimized formulation and is in good agreement with the data obtained from the in vitro release study. The initial faster release from PEG-free ATR-ISG formulation was due to rapid dissipation of solvent, NMP, which contained the solubilized drug while the later phase of drug release was controlled by polymer erosion and degradation.

\section{Conclusion}

The central composite optimization design has been successfully implemented to develop an optimized depot injectable ATR-ISG formulation with low in vitro initial drug release. SEM and FTIR confirmed the impact of PEG incorporation in minimizing the initial burst of the drug from the system and prolongation of its effect. The pharmacokinetic parameters of the optimized ATR-ISG formulation were markedly different from that of the corresponding PEG-free one with significant reduction in the initial drug burst in comparison with the marketed tablet. ATR as an example of drugs that are used for the treatment of chronic diseases could be formulated as an ISG system using biodegradable PLGA polymer. The developed ISG formulation showed a promising release control, mean residence time prolongation, and improvement of the relative bioavailability, which are more favorable for long-term hypolipidemic drug therapy.

\section{Disclosure}

The authors report no conflicts of interest in this work.

\section{References}

1. Chenite A, Chaput C, Wang D, et al. Novel injectable neutral solutions of chitosan form biodegradable gels in situ. Biomaterials. 2000;21(21):2155-2161.

2. Packhaeuser CB, Schnieders J, Oster CG, Kissel T. In situ forming parenteral drug delivery systems: an overview. Eur J Pharm Biopharm. 2004;58(2):445-455.
3. Ahmed TA. Approaches to develop PLGA based in situ gelling system with low initial burst. Pak J Pharm Sci. 2015;28(2): 657-665.

4. Rodrigues S, Cordeiro C, Seijo B, Remuñán-López C, Grenha A. Hybrid nanosystems based on natural polymers as protein carriers for respiratory delivery: stability and toxicological evaluation. Carbohydr Polym. 2015;123:369-380.

5. Kranz H, Brazeau GA, Napaporn J, Martin RL, Millard W, Bodmeier R. Myotoxicity studies of injectable biodegradable in-situ forming drug delivery systems. Int J Pharm. 2001;212(1):11-18.

6. Ahmed TA, Ibrahim HM, Ibrahim F, et al. Development of biodegradable in situ implant and microparticle injectable formulations for sustained delivery of haloperidol. J Pharm Sci. 2012;101(10): 3753-3762.

7. Ahmed TA, Ibrahim HM, Samy AM, Kaseem A, Nutan MTH, Hussain MD. Biodegradable injectable in situ implants and microparticles for sustained release of Montelukast: in vitro release, pharmacokinetics, and stability. AAPS PharmSciTech. 2014;15(3): 772-780.

8. Mallapragada SK, Peppas NA, Colombo P. Crystal dissolutioncontrolled release systems. II. Metronidazole release from semicrystalline poly(vinyl alcohol) systems. J Biomed Mater Res. 1997;36(1): $125-130$.

9. Pekarek KJ, Jacob JS, Mathiowitz E. Double-walled polymer microspheres for controlled drug release. Nature. 1994;367(6460): 258-260.

10. Graham P, Brodbeck K, McHugh A. Phase inversion dynamics of PLGA solutions related to drug delivery. J Control Release. 1999; 58(2):233-245.

11. Xin C, Lihong W, Qiuyuan L, Hongzhuo L. Injectable long-term control-released in situ gels of hydrochloric thiothixene for the treatment of schizophrenia: preparation, in vitro and in vivo evaluation. Int J Pharm. 2014;469(1):23-30.

12. Patel RB, Carlson AN, Solorio L, Exner AA. Characterization of formulation parameters affecting low molecular weight drug release from in situ forming drug delivery systems. $J$ Biomed Mater Res A. 2010;94(2):476-484.

13. Kempe S, Mäder K. In situ forming implants - an attractive formulation principle for parenteral depot formulations. J Control Release. 2012;161(2):668-679.

14. Ibrahim HM, Ahmed TA, Hussain MD, et al. Development of meloxicam in situ implant formulation by quality by design principle. Drug Dev Ind Pharm. 2013;9045:1-8.

15. Tan LP, Venkatraman SS, Sung PF, Wang XT. Effect of plasticization on heparin release from biodegradable matrices. Int $J$ Pharm. 2004;283(1-2):89-96.

16. Goldstein JL, Brown MS. Regulation of the mevalonate pathway. Nature. 1990;343(6257):425-430. 
17. Pekkanen J, Linn S, Heiss G, et al. Ten-year mortality from cardiovascular disease in relation to cholesterol level among men with and without preexisting cardiovascular disease. $N$ Engl J Med. 1990; 322(24):1700-1707.

18. Lennernäs H. Clinical pharmacokinetics of atorvastatin. Clin Pharmacokinet. 2003;42(13):1141-1160.

19. Wagner JG. Interpretation of percent dissolved-time plots derived from in vitro testing of conventional tablets and capsules. J Pharm Sci. 1969;58(10):1253-1257.

20. Desai S, Singh P, Simonelli A, Higuchi W. Investigation of factors influencing release of solid drug dispersed in inert matrices III. Quantitative studies involving the polyethylene plastic matrix. J Pharm Sci. 1966;55(11):1230-1234.

21. Higuchi T. Mechanism of sustained-action medication. Theoretical analysis of rate of release of solid drugs dispersed in solid matrices. J Pharm Sci. 1963;52(12):1145-1149.

22. Korsmeyer R, Gurny R, Doelker E, Buri P, Peppas N. Mechanisms of potassium chloride release from compressed, hydrophilic, polymeric matrices: effect of entrapped air. J Pharm Sci. 1983;15(1):1189-1191.

23. Ritger P, Peppas N. A simple equation for description of solute release. II. Fickian and anomalous release from swellable devices. J Control Release. 1987;5:37-42.

24. Kurakula M, Ahmed TA. Co-delivery of atorvastatin nanocrystals in PLGA based in situ gel for anti-hyperlipidemic efficacy. Curr Drug Deliv. Epub 2015 Nov 8.

25. Reagan-Shaw S, Nihal M, Ahmad N. Dose translation from animal to human studies revisited. FASEB J. 2008;22(3):659-661.

26. Gajula R, Pilli NR, Ravi VB, et al. Simultaneous determination of atorvastatin and aspirin in human plasma by LC-MS/MS: its pharmacokinetic application. Sci Pharm. 2012;80(4):923-940.

27. D'Souza SS, Faraj JA, DeLuca PP. A model-dependent approach to correlate accelerated with real-time release from biodegradable microspheres. AAPS PharmSciTech. 2005;6(4):E553-E564.

28. Wang L, Venkatraman S, Kleiner L. Drug release from injectable depots: two different in vitro mechanisms. J Control Release. 2004; 99(2):207-216.
29. Shive M, Anderson J. Biodegradation and biocompatibility of PLA and PLGA microspheres. Adv Drug Deliv Rev. 1997;28(1):5-24.

30. Jain RA. The manufacturing techniques of various drug loaded biodegradable poly(lactide-co-glycolide) (PLGA) devices. Biomaterials. 2000;21(23):2475-2490.

31. Fredenberg S, Wahlgren M, Reslow M, Axelsson A. The mechanisms of drug release in poly(lactic-co-glycolic acid)-based drug delivery systems - a review. Int J Pharm. 2011;415(1-2):34-52.

32. Tang Y, Singh J. Controlled delivery of aspirin: effect of aspirin on polymer degradation and in vitro release from PLGA based phase sensitive systems. Int J Pharm. 2008;357(1-2):119-125.

33. Elias-Al-Mamun M, Khan HA, Dewan I, Jalil R-U. In vitro study on tamsulosin release kinetics from biodegradable PLGA in situ implants. Pak J Pharm Sci. 2009;22(4):360-367.

34. Alimohammadi S, Salehi R, Amini N, Davaran S. Synthesis and physicochemical characterization of biodegradable PLGA-based magnetic nanoparticles containing amoxicilin. Bull Korean Chem Soc. 2012;33(10):3225-3232.

35. Deadman CM, Kellaway IW, Yasin M, Dickinson PA, Murdan S. An investigation into the influence of drug lipophilicity on the in vivo absorption profiles from subcutaneous microspheres and in situ forming depots. J Control Release. 2007;122(1):79-85.

36. Vintiloiu A, Lafleur M, Bastiat G, Leroux J-C. In situ-forming oleogel implant for rivastigmine delivery. Pharm Res. 2008;25(4):845-852.

37. Luan X, Bodmeier R. Modification of the tri-phasic drug release pattern of leuprolide acetate-loaded poly(lactide-co-glycolide) microparticles. Eur J Pharm Biopharm. 2006;63(2):205-214.

38. Grassi M, Grassi G. Mathematical modelling and controlled drug delivery: matrix systems. Curr Drug Deliv. 2005;2(1):97-116.

39. Shoaib MH, Tazeen J, Merchant HA, Yousuf RI. Evaluation of drug release kinetics from ibuprofen matrix tablets using HPMC. Pak J Pharm Sci. 2006;19(2):119-124.

\section{Publish your work in this journal}

Drug Design, Development and Therapy is an international, peerreviewed open-access journal that spans the spectrum of drug design and development through to clinical applications. Clinical outcomes, patient safety, and programs for the development and effective, safe, and sustained use of medicines are a feature of the journal, which

\section{Dovepress}

has also been accepted for indexing on PubMed Central. The manuscript management system is completely online and includes a very quick and fair peer-review system, which is all easy to use. Visit http://www.dovepress.com/testimonials.php to read real quotes from published authors. 